Sharif University of Technology
Scientia Iranica
Transactions E: Industrial Engineering
hCIENTIA

\title{
A mathematical model to optimize the reliability of the satellite attitude determination and control system
}

\author{
A. Mansouri ${ }^{a}$ and A. Alem-Tabriz ${ }^{\text {b,* }}$ \\ a. Department of Industrial Engineering, Faculty of Industrial and Mechanical Engineering, Qazvin Branch, Islamic Azad \\ University, Qazvin, Iran. \\ b. Department of Industrial Management, Management and Accounting Faculty, Shahid Beheshti University, Tehran, Iran.
}

Received 24 May 2020; received in revised form 18 August 2020; accepted 19 October 2020

\author{
KEYWORDS \\ Reliability; \\ Satellite; \\ Attitude \\ determination and \\ control system; \\ Redundancy \\ allocation problem; \\ Genetic algorithm.
}

\begin{abstract}
This study aims at the integration of redundancy allocation and optimization of failure rates. It is of necessity to simultaneously work with parallel allocation and increase the number of components in a subsystem to improve the reliability or availability of parallel-series systems and push it into practical and applicable domains. This research investigates the possibility of reducing failure rates of different components in the system. It is important to note that with a delicate analysis of the effects of each approach and the costs imposed on the system, the design problem data is formed. Considering that much effort made to improve the reliability of components ensures less redundancy allocation and vice versa, the optimization problem is performed to determine the exact number of redundancies and the exact level of improvement regarding complete failure rates. In this research, the satellite attitude determination and control system as well as the structure of the studied system and its components are introduced. Then, the reliability of this system is modeled and optimized using a mathematical approach based on a combination of reliability allocation and redundancy allocation.

(C) 2022 Sharif University of Technology. All rights reserved.
\end{abstract}

\section{Introduction}

Satellites are human-made devices that are deliberately sent into space to go around the earth or other planets. The importance of satellites for telecommunications and the study of terrestrial resources and research as well as military and espionage applications are growing. Part of the scientific and specialized research done in space-based laboratories has never been able to assume a practical dimension on earth. To control the safety of sensitive systems including those in nuclear power plants, chemical processes, and the astronaut system, it is necessary to establish ready-made safety

\footnotetext{
*. Corresponding author.

E-mail address: A-tabriz@sbu.ac.ir (A. Alem-Tabriz)
}

systems to complete online regulatory systems. Readyto-use systems can automatically operate to keep the system safe and prevent catastrophic outcomes under various conditions; however, ready-to-operate safety systems may not be able to perform the expected performance due to hidden failures. Therefore, it is very important to investigate these problems during testing and maintenance and to diagnose and resolve maintenance testing in accordance with the manufacturer's recommendations, which are generally cautious recommendations. In sensitive safety applications, the periodic maintenance period is sometimes determined by a supervisory team; for example, strict rules apply to intermittent maintenance to keep the uncertainty level low. In general, intermittent and frequent testing can increase the likelihood of damage to be detected. However, it may cause the system to crash faster due to the increased unnecessary costs imposed on resources. 
Therefore, logical and efficient testing of maintenance strategy is of great importance in ready-made safety systems.

In this research, upon identifying the structure of the satellite attitude determination and control system as well as the components used in it, the reliability of this system is analyzed and optimized. To this end, upon investigating the information related to the system under study, the assumptions of modeling and optimizing the system reliability are determined. In this regard, practical and systemic constraints are considered. Finally, the obtained results are reported.

In this paper, a joint reliability-redundancy optimization approach to satellite attitude determination and control system is presented. This model is developed on a multiple-mode system in which each mode has a specific reliability block diagram. Various subsystems of the system under study have an active, cold-ready or $k$-out-of- $n$ strategy, which are identified in the reliability block diagram of each mode. In this study, the percentage of improvements concerning the reduction of the component failure rates is considered, and the failure rate of the components used in the closed continuous interval may change.

\section{Literature review}

This section covers a number of studies that have been carried out on optimizing the reliability of satellites and examines each one of them. Satellite is a separate system with a limited communication, which is difficult to access after launch. This is the reason why it is very difficult to repair it if the system breaks down. Therefore, despite the risk of failure, the satellite system must perform well during its lifetime. Many factors form satellite threats including the environment, network problems, software errors, and so on [1]. Environmental threats include the effects of solar proton and electron damage caused by cosmic rays, leading to incorrect commands and inaccurate data [2]. Also, network threats can lead to command error caused by viruses, and Aplinks [3] software errors in satellites, in particular, are among the most important issues of long-lived systems and represent a priority. Increasing the lifespan of software causes disclosure of confidential resources and leads to gradual reduction of system performance [4].

Castet and Saleh [5] noted limited access to information and failure rates associated with various satellite subsystems, and they used the Kaplan-Meier estimator method to evaluate the system reliability. Not only did they use non-parametric methods for modeling, but they also used the Maximum Liklihood Estimation (MLE) method to use the Weibel distribution parameters to distribute life under different systems. In addition, they finally compared the results with each other. Eleven subsystems on the satellite focused on one of the most important achievements of the study.

Category 1: One year later, Castet and Saleh [6] considered the subsystems of a satellite to be multilayered components. In this case, each case is mentioned briefly. Nagiya and Ram carried out a study that used the Markov model to evaluate and optimize a satellite with specific information.

The assumptions of this research are given in the following [7]:

$\sqrt{ }$ Initially, all components of the system are intact;

$\sqrt{ }$ The satellite under study generally includes 8 states;

$\sqrt{ }$ All breakdown and repair rates are fixed over time;

$\sqrt{ }$ At one point, only one transfer from one state to another is allowed;

$\sqrt{ }$ The necessary equipment is available for repair;

$\sqrt{ }$ Each component after repair is like a new component;

$\sqrt{ }$ All satellite operating states are repairable.

In this study, the level of redundancy and failure rates in the satellite attitude determination and control system are optimized. In general, the issue of overallocation has been studied by many researchers. Fyffe et al. [8] were the first to present a mathematical model of the general problem of redundancy allocation. The objective of their proposed model was to maximize system reliability in terms of weight and cost constraints. They solved this problem through dynamic planning. Nakagawa and Miyazaki [9] presented a nonlinear planning problem with a solution to optimize reliability. In fact, by changing the example offered by Fyffe et al. [8], they solved the problem using the exact method of substituting constraints and demonstrated that in the case of multiple constraints, this approach outperformed the dynamic planning method. However, in order to increase the reliability, one or a set of the following measures can be performed. These measures can be implemented based on the assumptions and requirements of each system.

$\sqrt{ }$ Optimization of redundancy level;

$\sqrt{ }$ Optimization of the selection of component types used in the system;

$\sqrt{ }$ Optimization of existing technical activities in order to regulate failure and repair rates.

In the following, some conducted research studies on the optimization of the redundancy allocation problem are given in Table 1. 
Table 1. Assumption of mathematical optimization models in the redundancy allocation problem area.

\begin{tabular}{|c|c|c|c|c|c|c|c|c|c|}
\hline Authors & State & Element type & Algorithm & $\begin{array}{c}\text { Fuzzy } \\
\text { availability }\end{array}$ & $\begin{array}{c}\text { Fault } \\
\text { elements }\end{array}$ & $\begin{array}{l}\text { Penalty } \\
\text { function }\end{array}$ & Objective & $\begin{array}{c}\text { Parameter } \\
\text { setting }\end{array}$ & $\begin{array}{c}\text { Cost } \\
\text { discount } \\
\text { strategy }\end{array}$ \\
\hline Garg and Sharma [10] & Binary & Heterogeneous & Bee colony & No & Non-repairable & Yes & Single & No & No \\
\hline $\begin{array}{l}\text { Khalili-Damghani } \\
\text { et al. [11] }\end{array}$ & Binary & Heterogeneous & e-constraint & No & Non-repairable & No & Multiple & No & No \\
\hline Chambari et al. [12] & Binary & Heterogeneous & SA & No & Non-repairable & Yes & Single & No & No \\
\hline Gago et al. [13] & Binary & Heterogeneous & $\begin{array}{c}\text { Greedy, } \\
\text { walk back }\end{array}$ & No & Non-repairable & No & Single & No & No \\
\hline $\begin{array}{l}\text { Ebrahimipour } \\
\text { et al. [14] }\end{array}$ & Binary & Heterogeneous & $\begin{array}{c}\text { Fuzzy Inference } \\
\text { System (FIS) }\end{array}$ & No & Non-repairable & No & Single & No & No \\
\hline Liu et al. [15] & Multi-state & Heterogeneous & $\begin{array}{c}\text { Imperfect repair } \\
\text { model }\end{array}$ & Yes & Repairable & No & Single & No & No \\
\hline $\begin{array}{l}\text { Ding and } \\
\text { Lisnianski [16] }\end{array}$ & Multi-state & Heterogeneous & GA & No & Non-repairable & No & Single & No & No \\
\hline Ouzineb et al. [17] & Multi-state & Heterogeneous & GA & No & Non-repairable & No & Single & No & No \\
\hline $\begin{array}{l}\text { Sharma and } \\
\text { Agarwal [18] }\end{array}$ & Multi-state & Heterogeneous & $\mathrm{ACO}$ & No & Non-repairable & No & Single & No & No \\
\hline Ouzineb et al. [19] & Multi-state & Homogeneous & TS & No & Non-repairable & No & Single & No & AUD \\
\hline Levitin et al. [20] & Multi-state & Heterogeneous & GA & No & Non-repairable & No & Single & No & No \\
\hline Lins and Droguett [21] & Binary & Heterogeneous & GA & No & Repairable & No & Multiple & No & No \\
\hline Lins and Droguett [22] & Binary & Heterogeneous & $\mathrm{ACO}$ & No & Repairable & No & Multiple & No & No \\
\hline Maatouk et al. [23] & Multi-state & Heterogeneous & GA & No & Repairable & No & Single & No & No \\
\hline Garg et al. [24] & Binary & Heterogeneous & GA & No & Non-repairable & No & Multiple & No & No \\
\hline $\begin{array}{l}\text { Ebrahimipour and } \\
\text { Sheikhalishahi [25] }\end{array}$ & Binary & Heterogeneous & PSO & Yes & Non-repairable & No & Multiple & No & AUD \\
\hline Miriha et al. [26] & Binary & Heterogeneous & $\begin{array}{l}\text { NSGA-II } \\
\text { MOEA/D }\end{array}$ & No & Non-repairable & Yes & Multiple & Taguchi & No \\
\hline Mousavi et al. [27] & Multi-state & Homogeneous & CE-NRGA & Yes & Non-repairable & Yes & Multiple & Taguchi & $\begin{array}{l}\text { AUD and } \\
\text { IQD }\end{array}$ \\
\hline Zaretalab et al. [28] & Multi-state & Homogeneous & MOSA & No & Non-repairable & Yes & Multiple & No & No \\
\hline
\end{tabular}

\section{Satellite attitude determination and control system}

This study investigates the satellite attitude determination and control system. High efficiency, reliability, and health are the most important criteria in the engineering of space systems. Reliability refers to the possibility of the system working properly over a period of time. The first and most important step in preventing a system from malfunctioning is to detect failure in that system. Because, in this case, there is enough time to prevent problems in the system. Satellites are examples of self-contained, important, and costly space systems that are sent on relatively long-term missions. One of the most important parts of satellite that is directly related to health is the subsystem of satellite attitude determination and control system. The task of this subsystem is to determine and control the situation in space and neutralize the disturbing environmental disturbances and torques on the satellite. Therefore, this subsystem is always known as a subject of study that identifies breakdowns because improving the subsystem reliability of satellite attitude determination and control system directly affects the reliability of the satellite in space. To design a subsystem of satellite attitude determination and control system, in most cases, three reaction wheels are used in line with three main axes of the satellite. Each of these actuators has an electric motor and a heavy disk. With the application of current to the electric motor, torque is produced, which changes the speed and the angles of the motor axis. The opposite direction is produced. Changing the speed of the reaction wheel by applying the necessary control algorithm to the motors motivates them to reach the required speed from zero. After producing the necessary torque, the motor shuts off again. This change in speed leads to the generation of the required tower radiation in order 


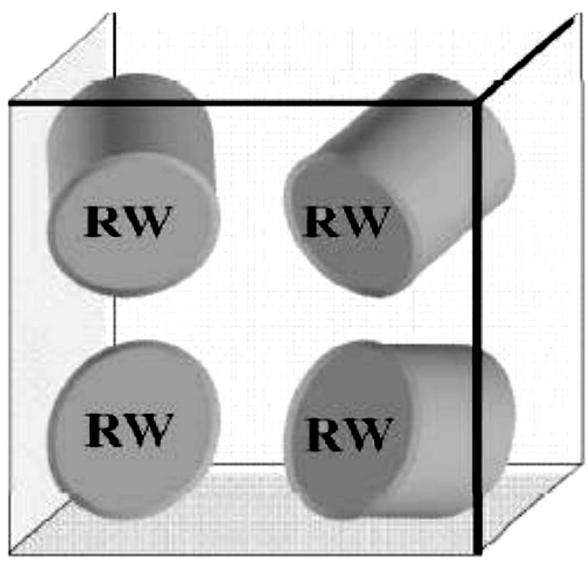

Figure 1. A schematic view of reaction wheel.

to achieve the desired state of the satellite. In order to promote reliability, a reaction or spare reaction cycle is used under the systems based on the reaction wheel. According to Figure 1, this actuator is located next to the main reaction cycle and as soon as one of the wheels fails or irreparable damage occurs in them, the spare reaction wheel will replace the defective cycle in the system. In general, the modules of the system studied in this research are shown in Figure 2.

\section{Problem statement}

In this section, the satellite attitude determination and control system as well as the structure of the system under study and its components are introduced. Then, the reliability of this system is modeled and optimized using the mathematical approach based on a combination of reliability allocation and redundancy allocation. In the following, the components, structure, and functions of the satellite attitude determination and control system are introduced.

\subsection{Functions of attitude determination and control system}

The functions of the attitude determination and control system include the following:

- Adjust the satellite to the desired direction despite external disturbance torques;

- Determine the attitude of satellites using sensors;

- Switch the situation by actuators;

- Set the satellite orientation for the mission.

The sub-system used for determining and controlling the situation to perform each of its tasks in the form of control modes and obtaining the required attitude in each mode consists of two parts: determination and control. The attitude determination section includes sensors and attitude determination algorithms, and the attitude control section includes actuators and attitude control algorithms.

\subsection{Components of attitude determination and control system}

The attitude determination and control system consists of several parts that are given in the following:

- Electronic Control Unit (ECU)

- Interface (Int)

- Sun Sensors (SS)

- Magnetic Sensor (MM)

- Gyro sensor (Gyro)

- Star Sensor (ST)

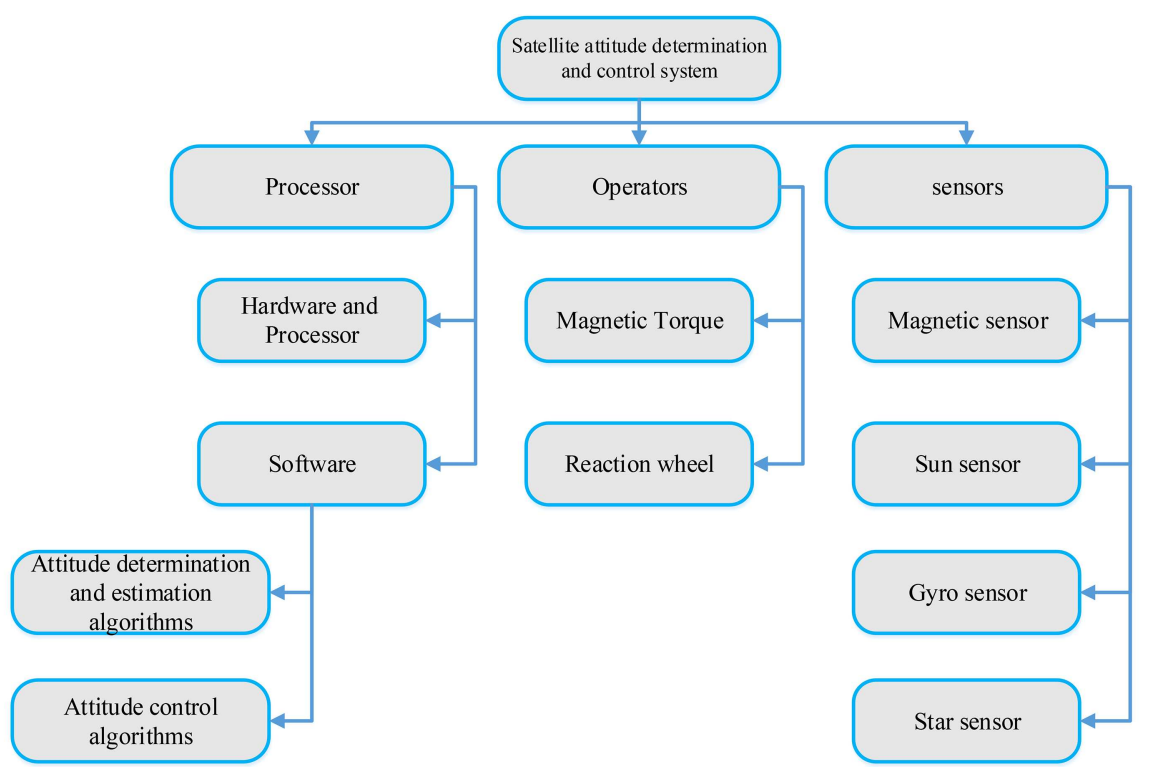

Figure 2. The modules of the satellite attitude determination and control system. 
- Magnetic Torquer (MT)

- Reaction Wheel (RW)

\subsection{Modes of attitude determination and control system}

In general, the satellite attitude determination and control system analyzed in this research has five different functional modes: DE tumbling mode, coarse pointing mode, fine pointing mode, sun pointing mode, and safe mode. Reliability block diagram of these modes in Figures 3 to 7 is provided.

In these reliability block diagrams, subsystems marked by gate $\mathrm{s}$ (before the subsystem) are coldstandby. Values of $K$ and $N$ for $k$-out-of- $n$ subsystems are specified. For example, the reaction wheel subsystem in the coarse pointing mode is 2-out-of4. Subsystems with active redundancy are unmarked. Other subsystems are series in type.

\subsection{The parameters and variables of the problem}

Table 2 introduces the parameters while Table 3 introduces the variables of optimizing the reliability of satellite attitude determination and control problem.

\subsection{Assumptions}

- The function of the components used in the system is independent of each other;

- The failure of components used in the system is independent of each other;

- The components of the system under study are binary;

- The components cannot be repaired and returned to the system after failure;

- The parameters related to the cost and weight of the components in the system are deterministic and definite;

- The system has maximum cost and maximum weight allowed for the components used in it;

- The system has different functional modes;

- The various subsystems of the system under study are active, cold-ready, or $k$-out-of- $n$;

- The failure rate of the components used in the system is constant;

- The lifetime distribution of the components used in the system is exponential;

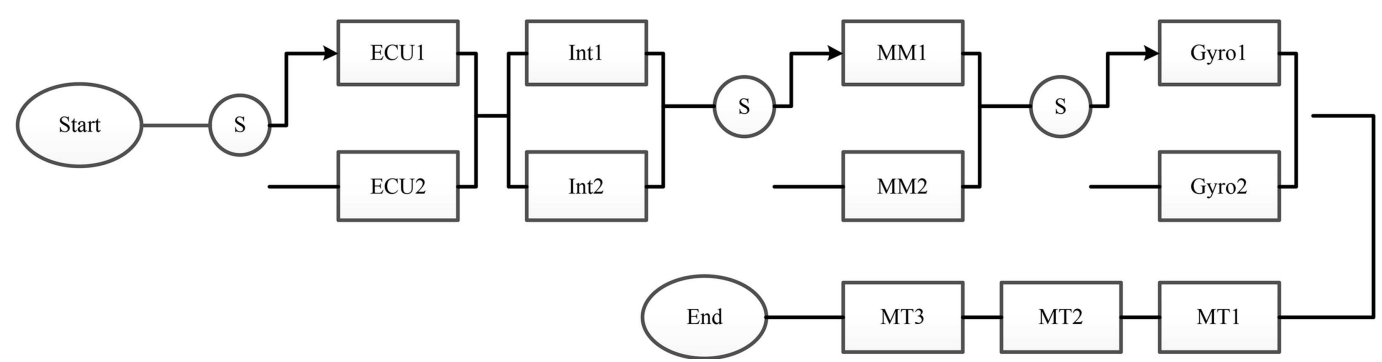

Figure 3. The reliability block diagram of the satellite attitude determination and control system under DE tumbling mode.

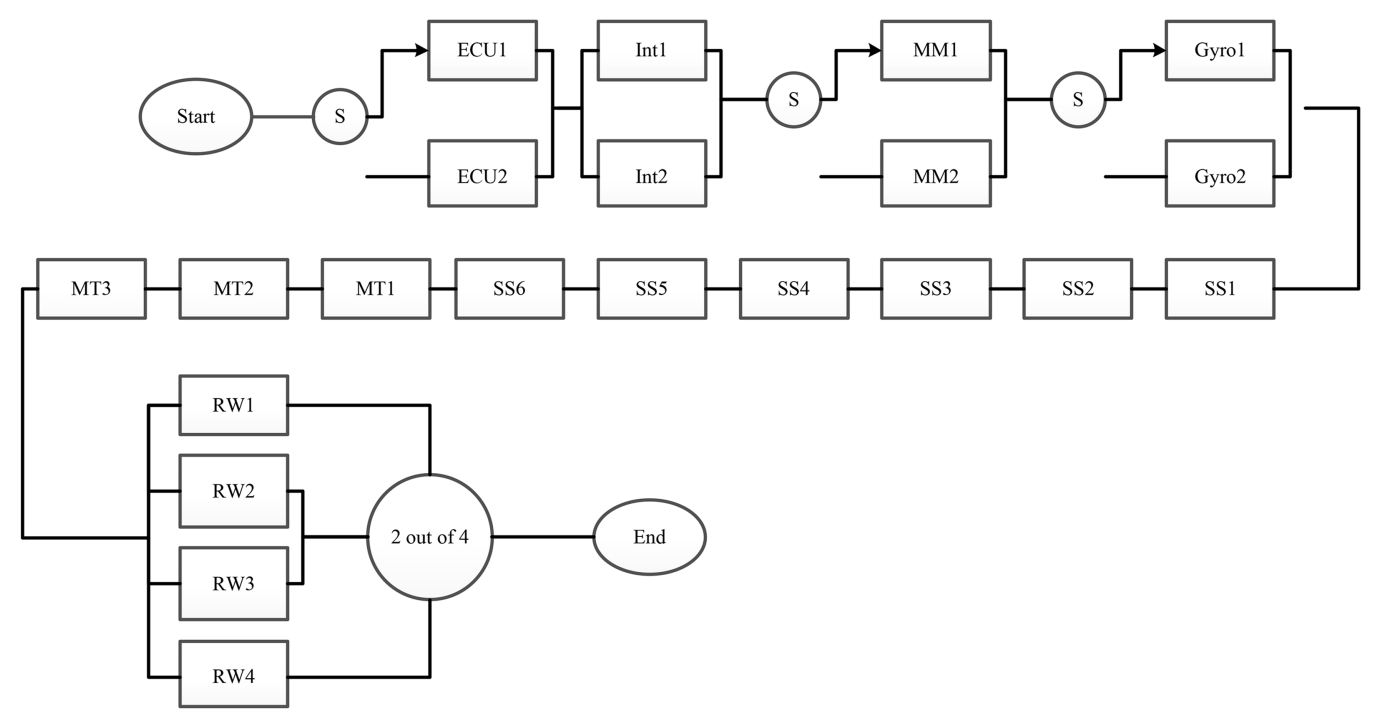

Figure 4. The reliability block diagram of the satellite attitude determination and control system under coarse pointing mode. 


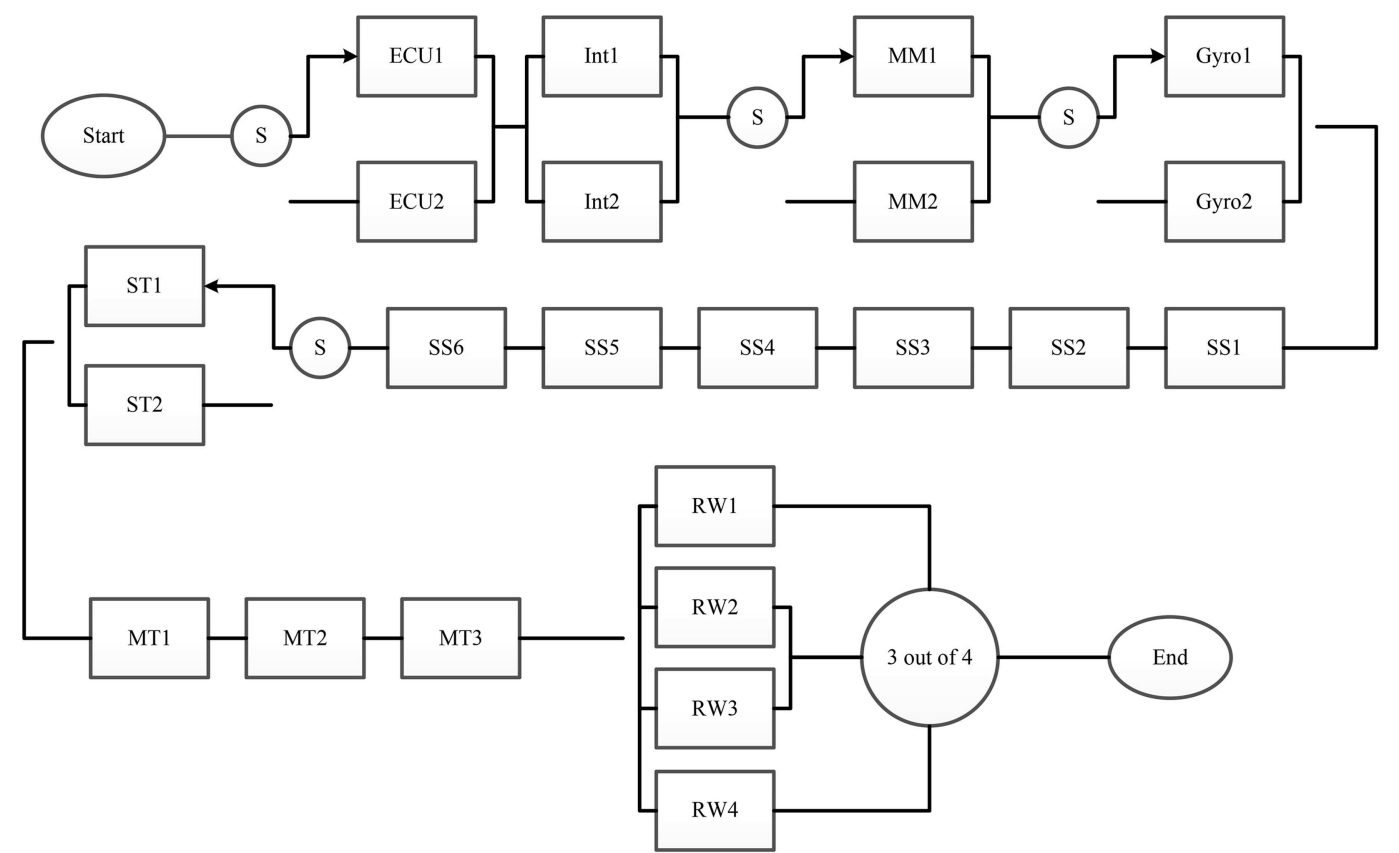

Figure 5. The reliability block diagram of the satellite attitude determination and control system under fine pointing mode.
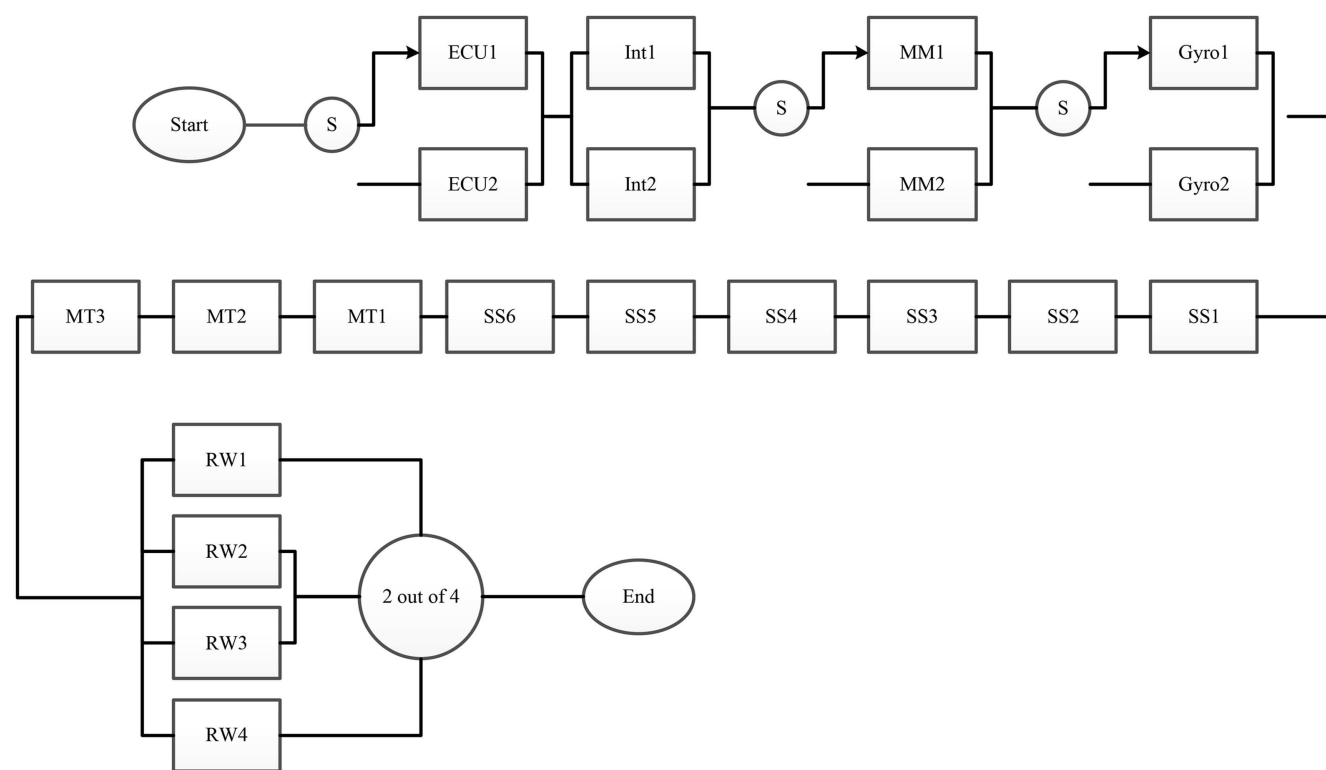

Figure 6. The reliability block diagram of the satellite attitude determination and control system under sun pointing mode.

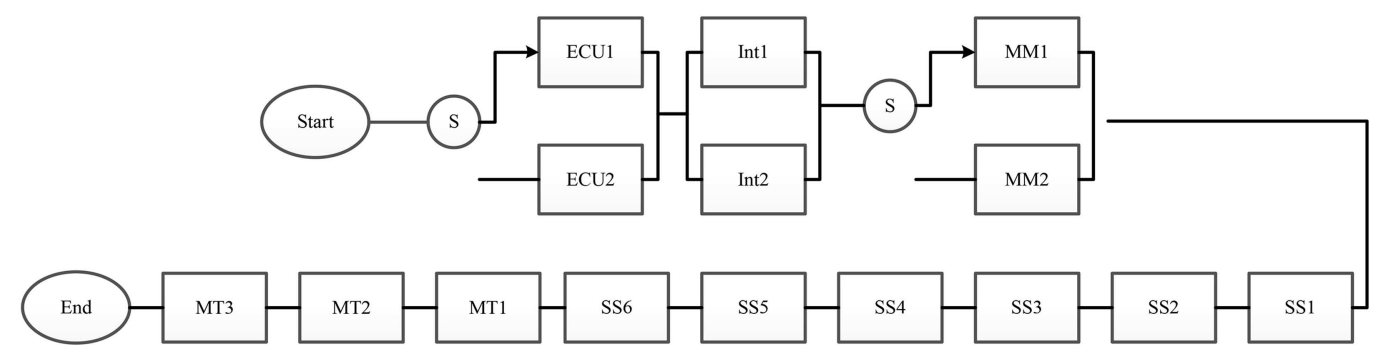

Figure 7. The reliability block diagram of the satellite attitude determination and control system under safe mode. 
Table 2. Reliability optimization problem parameters of attitude determination and control system.

\begin{tabular}{|c|c|}
\hline Parameters & Descriptions \\
\hline$t$ & System mission time \\
\hline$\rho_{E C U}$ & Safe operation possibility of the switch for the electric control unit \\
\hline$\rho_{M M}$ & Safe operation possibility of the switch for magnetic sensor \\
\hline$\rho_{\text {Gyro }}$ & Safe operation possibility of the switch for the gyro sensor \\
\hline$\rho_{S T}$ & Safe operation possibility of the switch for the star sensor \\
\hline$\lambda_{E C U}^{\max }$ & Maximum electronic control unit failure rate \\
\hline$\lambda_{E C U}^{\min }$ & Minimum electronic control unit failure rate \\
\hline$\lambda_{\text {Int }}^{\max }$ & Maximum interface failure rate \\
\hline$\lambda_{\text {Int }}^{\min }$ & Minimum interface failure rate \\
\hline$\lambda_{M M}^{\max }$ & Maximum magnetic sensor failure rate \\
\hline$\lambda_{M M}^{\min }$ & Minimum magnetic sensor failure rate \\
\hline$\lambda_{\text {Gyro }}^{\max }$ & Maximum gyro sensor failure rate \\
\hline$\lambda_{G y r o}^{\min }$ & Minimum gyro sensor failure rate \\
\hline$\lambda_{S S}^{\max }$ & Maximum sun sensors failure rate \\
\hline$\lambda_{S S}^{\min }$ & Minimum sun sensors failure rate \\
\hline$\lambda_{S T}^{\max }$ & Maximum star sensor failure rate \\
\hline$\lambda_{S T}^{\min }$ & Minimum star sensor failure rate \\
\hline$\lambda_{M T}^{\max }$ & Maximum magnetic torquer failure rate \\
\hline$\lambda_{M T}^{\min }$ & Minimum magnetic torquer failure rate \\
\hline$\lambda_{R W}^{\max }$ & Maximum reaction wheel failure rate \\
\hline$\lambda_{R W}^{\min }$ & Minimum reaction wheel failure rate \\
\hline$U_{E C U}$ & Maximum number of redundant electronic control units in the system \\
\hline$L_{E C U}$ & Minimum number of redundant electronic control units in the system \\
\hline$U_{\text {Int }}$ & Maximum number of redundant interfaces in the system \\
\hline$L_{\text {Int }}$ & Minimum number of redundant interfaces in the system \\
\hline$U_{M M}$ & Maximum number of redundant magnetic sensors in the system \\
\hline$L_{M M}$ & Minimum number of redundant magnetic sensors in the system \\
\hline$U_{\text {Gyro }}$ & Maximum number of redundant gyro sensors in the system \\
\hline$L_{\text {Gyro }}$ & Minimum number of redundant gyro sensors in the system \\
\hline$U_{S T}$ & Maximum number of redundant star sensors in the system \\
\hline$L_{S T}$ & Minimum number of redundant star sensors in the system \\
\hline$U_{R W}$ & Maximum number of redundant reactive wheels in the system \\
\hline$L_{R W}$ & Minimum number of redundant reactive wheels in the system \\
\hline$C_{E C U}$ & The cost of each electric control unit \\
\hline$W_{E C U}$ & The weight of each electric control unit \\
\hline$D_{E C U}$ & $\begin{array}{l}\text { Cost of one-percent improvement in reducing the failure rate of the electric control unit } \\
\text { compared to the maximum possible failure rate }\end{array}$ \\
\hline$C_{\text {Int }}$ & The cost of each interface \\
\hline$W_{\text {Int }}$ & The weight of each interface \\
\hline$D_{\text {Int }}$ & $\begin{array}{l}\text { The cost of one-percent improvement in reducing interface failure rate compared to } \\
\text { the maximum possible failure rate }\end{array}$ \\
\hline$C_{M M}$ & The cost of each magnetic sensor \\
\hline$W_{M M}$ & The weight of each magnetic sensor \\
\hline$D_{M M}$ & $\begin{array}{l}\text { The cost of one-percent improvement in reducing the magnetic sensor failure rate compared to } \\
\text { the maximum possible failure rate }\end{array}$ \\
\hline
\end{tabular}


Table 2. Reliability optimization problem parameters of attitude determination and control system (continued).

\begin{tabular}{ll}
\hline Parameters & \multicolumn{1}{c}{ Descriptions } \\
\hline$C_{G y r o}$ & The cost of each gyro sensor \\
$W_{G y r o}$ & The weight of each gyro sensor \\
$D_{G y r o}$ & The cost of one-percent improvement in reducing the gyro sensor failure rate compared to \\
& the maximum possible failure rate \\
$D_{S S}$ & The cost of one-percent improvement in reducing the solar sensor failure rate compared to \\
$C_{S T}$ & the maximum possible failure rate \\
$W_{S T}$ & The cost of each star sensor \\
$D_{S T}$ & The weight of each star sensor \\
$D_{M T}$ & The cost of one-percent improvement in reducing the star sensor failure rate compared to \\
$C_{R W}$ & the maximum possible failure rate \\
$W_{R W}$ & The cost of one-percent improvement in reducing the magnetic torquer failure rate compared to \\
$D_{R W}$ & The maximum possible failure rate \\
$C_{\max }$ & The weight of each wheel reacts \\
$W_{\max }$ & The one-percent improvement in reducing the reaction wheel failure rate compared to \\
$N_{S S}$ & the maximum possible failure rate \\
$N_{M T}$ & Maximum allowable cost
\end{tabular}

- The percentage of improvements with regard to reducing component failure rates is an integer number;

- In systems where the components are cold-standby, there is a possibility that the switch will fail;

- The failure rate of the components in a closed continuous interval may change.

\subsection{Mathematical modeling}

Based on the structure of the attitude determination and control system under each of the functional modes and, also, based on the arrangement of components according to the reliability block diagrams presented in Sections 3 and 4, the reliability of the system under each functional mode is calculated. According to the reliability block diagram provided, the structure of the attitude determination and control system in various functional modes of the hybrid structure includes active, cold-standby, and $k$-out-of- $n$. In this section, we first model the reliability level of the attitude determination and control system in each of the functional modes under conditions where the components used in the system follow any desired life distribution. Then, by placing the probability density function and the reliability of the exponential distribution in Eqs. (1) to $(5)$, the reliability of the system is presented in the functional modes of de tumbling, coarse pointing, fine pointing, sun pointing, and safe.

$$
\begin{aligned}
R_{D}(t)= & {\left[R_{E C U}(t)+\sum_{j=1}^{N_{E C U}-1} \rho_{E C U}(t)\right.} \\
& \left.\int_{0}^{t} f_{E C U}(u)^{(j)} R_{E C U}(t-u) d u\right] \\
& \times\left[1-\left(1-R_{\text {Int }}(t)\right)^{N_{I n t}}\right] \\
& \times\left[R_{M M}(t)+\sum_{j=1}^{N_{M M}-1} \rho_{M M}(t)\right. \\
& \left.\int_{0}^{t} f_{M M}(u)^{(j)} R_{M M}(t-u) d u\right] \\
& \times\left[R_{G y r o}(t)+\sum_{j=1}^{N_{G y r o}-1} \rho_{G y r o}(t)\right.
\end{aligned}
$$


Table 3. Reliability optimization problem variables of attitude determination and control system.

\begin{tabular}{|c|c|}
\hline Variables & Descriptions \\
\hline$R_{D}(t)$ & Reliability of the attitude determination and control system under the de tumbling functional mode \\
\hline$R_{C}(t)$ & Reliability of the attitude determination and control system under the coarse pointing functional mode \\
\hline$R_{F}(t)$ & Reliability of the attitude determination and control system under the fine pointing functional mode \\
\hline$R_{S U}(t)$ & Reliability of the attitude determination and control system under the sun pointing functional mode \\
\hline$R_{S E}(t)$ & Reliability of the attitude determination and control system under the safe functional mode \\
\hline$N_{E C U}$ & Number of electronic control units in the system \\
\hline$\lambda_{E C U}$ & The failure rate of each electronic control unit in the system \\
\hline$x_{E C U}$ & $\begin{array}{l}\text { Percentage of improvement in reducing the electric control unit failure rate compared to the } \\
\text { maximum possible failure rate }\end{array}$ \\
\hline$N_{\text {Int }}$ & The number of interfaces in the system \\
\hline$\lambda_{\text {Int }}$ & The failure rate of each interface in the system \\
\hline$x_{\text {Int }}$ & $\begin{array}{l}\text { Percentage of improvement in reducing the interface failure rate compared to } \\
\text { the maximum possible failure rate }\end{array}$ \\
\hline$N_{M M}$ & The number of magnetic sensors in the system \\
\hline$\lambda_{M M}$ & The failure rate of any magnetic sensor in the system \\
\hline$x_{M M}$ & $\begin{array}{l}\text { The percentage improvement in reducing the magnetic sensor failure rate compared to } \\
\text { the maximum possible failure rate }\end{array}$ \\
\hline$N_{\text {Gyro }}$ & The number of gyro sensors in the system \\
\hline$\lambda_{\text {Gyro }}$ & Failure rate of any gyro sensor located in the system \\
\hline$x_{\text {Gyro }}$ & $\begin{array}{l}\text { Percentage of improvements in reducing the gyro sensor failure rate compared to } \\
\text { the maximum possible failure rate }\end{array}$ \\
\hline$\lambda_{S S}$ & The failure rate of each solar sensor in the system \\
\hline$x_{S S}$ & $\begin{array}{l}\text { Percentage of improvement in reducing solar sensor failure rate compared to } \\
\text { the maximum possible failure rate }\end{array}$ \\
\hline$N_{S T}$ & The number of star sensors in the system \\
\hline$\lambda_{S T}$ & The failure rate of each star sensor in the system \\
\hline$x_{S T}$ & $\begin{array}{l}\text { Percentage of improvement in reducing star sensor failure rate compared to } \\
\text { the maximum possible failure rate }\end{array}$ \\
\hline$\lambda_{M T}$ & The failure rate of each magnetic torquer located in the system \\
\hline$x_{M T}$ & $\begin{array}{l}\text { Percentage of improvement in reducing magnetic torquer failure rate compared to } \\
\text { the maximum possible failure rate }\end{array}$ \\
\hline$N_{R W}$ & The number of reaction wheels in the system \\
\hline$\lambda_{R W}$ & The failure rate of each reaction wheel is located in the system \\
\hline$x_{R W}$ & $\begin{array}{l}\text { Percentage of improvement in reducing wheel failure rate reaction to } \\
\text { the maximum possible failure rate }\end{array}$ \\
\hline
\end{tabular}

$$
\begin{aligned}
& \left.\int_{0}^{t} f_{\text {Gyro }}(u)^{(j)} R_{\text {Gyro }}(t-u) d u\right] \\
& \times\left[R_{M T}(t)^{N_{M T}}\right], \\
R_{C}(t)= & {\left[R_{E C U}(t)+\sum_{j=1}^{N_{E C U}-1} \rho_{E C U}(t)\right.}
\end{aligned}
$$$$
\left.\int_{0}^{t} f_{E C U}(u)^{(j)} R_{E C U}(t-u) d u\right]
$$$$
\begin{aligned}
& \times\left[1-\left(1-R_{\text {Int }}(t)\right)^{N_{\text {Int }}}\right] \\
& \times\left[R_{M M}(t)+\sum_{j=1}^{N_{M M}-1} \rho_{M M}(t)\right.
\end{aligned}
$$ 


$$
\begin{aligned}
& \left.\int_{0}^{t} f_{M M}(u)^{(j)} R_{M M}(t-u) d u\right] \\
& \left.\int_{0}^{t} f_{S T}(u)^{(j)} R_{S T}(t-u) d u\right] \\
& \times\left[R_{\text {Gyro }}(t)+\sum_{j=1}^{N_{\text {Gyro }}-1} \rho_{\text {Gyro }}(t)\right. \\
& \left.\int_{0}^{t} f_{\text {Gyro }}(u)^{(j)} R_{\text {Gyro }}(t-u) d u\right] \\
& \times\left[R_{S S}(t)^{N_{S S}}\right] \times\left[R_{M T}(t)^{N_{M T}}\right] \\
& \times\left[\sum_{k=2}^{N_{R W}}\left(\begin{array}{c}
N_{R W} \\
k
\end{array}\right) R_{R W}(t)^{k}\right. \\
& \left.\left(1-R_{R W}(t)\right)^{N_{R W}-k}\right], \\
& R_{F}(t)=\left[R_{E C U}(t)+\sum_{j=1}^{N_{E C U}-1} \rho_{E C U}(t)\right. \\
& \left.\int_{0}^{t} f_{E C U}(u)^{(j)} R_{E C U}(t-u) d u\right] \\
& \times\left[R_{M T}(t)^{N_{M T}}\right] \times\left[\sum_{k=3}^{N_{R W}}\left(\begin{array}{c}
N_{R W} \\
k
\end{array}\right)\right. \\
& \left.R_{R W}(t)^{k}\left(1-R_{R W}(t)\right)^{N_{R W}-k}\right] \\
& R_{S U}(t)=\left[R_{E C U}(t)+\sum_{j=1}^{N_{E C U}-1} \rho_{E C U}(t)\right. \\
& \left.\int_{0}^{t} f_{E C U}(u)^{(j)} R_{E C U}(t-u) d u\right] \\
& \times\left[1-\left(1-R_{\text {Int }}(t)\right)^{N_{I n t}}\right] \\
& \times\left[R_{M M}(t)+\sum_{j=1}^{N_{M M}-1} \rho_{M M}(t)\right. \\
& \left.\int_{0}^{t} f_{M M}(u)^{(j)} R_{M M}(t-u) d u\right] \\
& \times\left[R_{\text {Gyro }}(t)+\sum_{j=1}^{N_{\text {Gyro }}-1} \rho_{\text {Gyro }}(t)\right. \\
& \times\left[1-\left(1-R_{\text {Int }}(t)\right)^{N_{I n t}}\right] \\
& \times\left[R_{M M}(t)+\sum_{j=1}^{N_{M M}-1} \rho_{M M}(t)\right. \\
& \left.\int_{0}^{t} f_{\text {Gyro }}(u)^{(j)} R_{\text {Gyro }}(t-u) d u\right] \\
& \times\left[R_{S S}(t)^{N_{S S}}\right] \times\left[R_{M T}(t)^{N_{M T}}\right] \\
& \left.\int_{0}^{t} f_{M M}(u)^{(j)} R_{M M}(t-u) d u\right] \\
& \times\left[\sum_{k=2}^{N_{R W}}\left(\begin{array}{c}
N_{R W} \\
k
\end{array}\right)\right. \\
& \left.R_{R W}(t)^{k}\left(1-R_{R W}(t)\right)^{N_{R W}-k}\right] \\
& R_{S E}(t)=\left[R_{E C U}(t)+\sum_{j=1}^{N_{E C U}-1} \rho_{E C U}(t)\right. \\
& \left.\int_{0}^{t} f_{\text {Gyro }}(u)^{(j)} R_{\text {Gyro }}(t-u) d u\right] \\
& \times\left[R_{S S}(t)^{N_{S S}}\right] \times\left[R_{S T}(t)+\sum_{j=1}^{N_{S T}-1} \rho_{S T}(t)\right. \\
& \begin{array}{l}
\left.\int_{0}^{t} f_{E C U}(u)^{(j)} R_{E C U}(t-u) d u\right] \\
\times\left[1-\left(1-R_{\text {Int }}(t)\right)^{N_{\text {Int }}}\right]
\end{array} \\
& \times\left[R_{\text {Gyro }}(t)+\sum_{j=1}^{N_{\text {Gyro }}-1} \rho_{\text {Gyro }}(t)\right.
\end{aligned}
$$




$$
\begin{aligned}
& \times\left[R_{M M}(t)+\sum_{j=1}^{N_{M M}-1} \rho_{M M}(t)\right. \\
& \left.\int_{0}^{t} f_{M M}(u)^{(j)} R_{M M}(t-u) d u\right] \\
& \times\left[R_{S S}(t)^{N_{S S}}\right] \times\left[R_{M T}(t)^{N_{M T}}\right] .
\end{aligned}
$$

Now, under the assumptions provided in Sections 4 and 5 , according to the information of the system for determining and controlling the attitude of the satellite base as well as evaluating the reliability of the system under different functional modes, the mathematical optimization model allocates reliability. In addition, the redundancy allocation for the system under study is presented below. In this model, the reliability of each functional mode based on the arrangement of components and system structure in the block of other names provided for each of these modes is calculated. Also, the failure rate of the components used in the fixed system is assumed. It is used in the display system; thus, the probability density function and the reliability function of the display distribution are placed in the set of equations presented to calculate the reliability of the system under different functional modes.

$\max \left\{\min \left\{R_{D}, R_{C}, R_{F}, R_{S U}, R_{S E}\right\}\right\}$,

s.t.:

$$
\begin{aligned}
R_{D}(t)= & {\left[\exp \left(-\lambda_{E C U} \cdot t\right)+\rho_{E C U}(t) \cdot \exp \left(-\lambda_{E C U} \cdot t\right)\right.} \\
& \left.\cdot \sum_{j=1}^{N_{E C U}-1} \frac{\left(\lambda_{E C U} \cdot t\right)^{j}}{j !}\right] \\
& \times\left[1-\left(1-\exp \left(-\lambda_{\text {Int }} \cdot t\right)\right)^{N_{\text {Int }}}\right] \\
& \times\left[\exp \left(-\lambda_{M M} \cdot t\right)+\rho_{M M}(t) \cdot \exp \left(-\lambda_{M M} \cdot t\right)\right. \\
& \left.\cdot \sum_{j=1}^{N_{M M}-1} \frac{\left(\lambda_{M M} \cdot t\right)^{j}}{j !}\right] \\
& \times\left[\exp \left(-\lambda_{G y r o} \cdot t\right)+\rho_{G y r o}(t) \cdot \exp \left(-\lambda_{G y r o} \cdot t\right)\right. \\
& \times\left[\exp \left(-\lambda_{M T} \cdot t\right)^{N_{M T}}\right], \\
& \left.\cdot \sum_{j=1}^{N_{G y r o}-1} \frac{\left(\lambda_{G y r o} \cdot t\right)^{j}}{j !}\right]
\end{aligned}
$$

$$
\begin{aligned}
& R_{C}(t)=\left[\exp \left(-\lambda_{E C U} \cdot t\right)+\rho_{E C U}(t) \cdot \exp \left(-\lambda_{E C U} \cdot t\right)\right. \\
& \left.\sum_{j=1}^{N_{E C U}-1} \frac{\left(\lambda_{E C U} \cdot t\right)^{j}}{j !}\right] \\
& \times\left[1-\left(1-\exp \left(-\lambda_{\text {Int }} . t\right)\right)^{N_{\text {Int }}}\right] \\
& \times\left[\exp \left(-\lambda_{M M} \cdot t\right)+\rho_{M M}(t) \cdot \exp \left(-\lambda_{M M} \cdot t\right)\right. \\
& \left.\cdot \sum_{j=1}^{N_{M M}-1} \frac{\left(\lambda_{M M} \cdot t\right)^{j}}{j !}\right] \times\left[\exp \left(-\lambda_{\text {Gyro }} \cdot t\right)\right. \\
& +\rho_{\text {Gyro }}(t) \cdot \exp \left(-\lambda_{\text {Gyro }} . t\right) \\
& \left.\cdot \sum_{j=1}^{N_{\text {Gyro }}-1} \frac{\left(\lambda_{\text {Gyro }} \cdot t\right)^{j}}{j !}\right] \times\left[\exp \left(-\lambda_{S S} \cdot t\right)^{N_{S S}}\right] \\
& \times\left[\exp \left(-\lambda_{M T} \cdot t\right)^{N_{M T}}\right] \\
& \times\left[\sum_{k=2}^{N_{R W}}\left(\begin{array}{c}
N_{R W} \\
k
\end{array}\right) \exp \left(-\lambda_{R W} \cdot t\right)^{k}\right. \\
& \left.\left(1-\exp \left(-\lambda_{R W} \cdot t\right)\right)^{N_{R W}-k}\right] \text {, }
\end{aligned}
$$$$
R_{F}(t)=\left[\exp \left(-\lambda_{E C U} \cdot t\right)+\rho_{E C U}(t) \cdot \exp \left(-\lambda_{E C U} \cdot t\right)\right.
$$$$
\left.\sum_{j=1}^{N_{E C U}-1} \frac{\left(\lambda_{E C U} \cdot t\right)^{j}}{j !}\right]
$$$$
\times\left[1-\left(1-\exp \left(-\lambda_{\text {Int }} . t\right)\right)^{N_{\text {Int }}}\right]
$$$$
\times\left[\exp \left(-\lambda_{M M} \cdot t\right)+\rho_{M M}(t) \cdot \exp \left(-\lambda_{M M} \cdot t\right)\right.
$$$$
\left.\sum_{j=1}^{N_{M M}-1} \frac{\left(\lambda_{M M} \cdot t\right)^{j}}{j !}\right] \times\left[\exp \left(-\lambda_{G y r o} . t\right)\right.
$$$$
+\rho_{\text {Gyro }}(t) \cdot \exp \left(-\lambda_{\text {Gyro }} . t\right)
$$$$
\left.\cdot \sum_{j=1}^{N_{\text {Gyro }}-1} \frac{\left(\lambda_{\text {Gyro }} \cdot t\right)^{j}}{j !}\right] \times\left[\exp \left(-\lambda_{S S} \cdot t\right)^{N_{S S}}\right]
$$$$
\times\left[\exp \left(-\lambda_{S T} \cdot t\right)+\rho_{S T}(t) \cdot \exp \left(-\lambda_{S T} \cdot t\right)\right.
$$ 


$$
\begin{aligned}
& \left.\cdot \sum_{j=1}^{N_{S T}-1} \frac{\left(\lambda_{S T} \cdot t\right)^{j}}{j !}\right] \times\left[\exp \left(-\lambda_{M T} \cdot t\right)^{N_{M T}}\right] \\
& \times\left[\sum_{k=3}^{N_{R W}}\left(\begin{array}{c}
N_{R W} \\
k
\end{array}\right) \exp \left(-\lambda_{R W} \cdot t\right)^{k}\right. \\
& \left.\left(1-\exp \left(-\lambda_{R W} \cdot t\right)\right)^{N_{R W}-k}\right]
\end{aligned}
$$$$
R_{S U}(t)=\left[\exp \left(-\lambda_{E C U} \cdot t\right)+\rho_{E C U}(t) \cdot \exp \left(-\lambda_{E C U} \cdot t\right)\right.
$$$$
\left.\cdot \sum_{j=1}^{N_{E C U}-1} \frac{\left(\lambda_{E C U} \cdot t\right)^{j}}{j !}\right] \times
$$$$
\left[1-\left(1-\exp \left(-\lambda_{\text {Int }} \cdot t\right)\right)^{N_{I n t}}\right]
$$$$
\times\left[\exp \left(-\lambda_{M M} \cdot t\right)+\rho_{M M}(t) \cdot \exp \left(-\lambda_{M M} \cdot t\right)\right.
$$$$
\left.\cdot \sum_{j=1}^{N_{M M}-1} \frac{\left(\lambda_{M M} \cdot t\right)^{j}}{j !}\right] \times\left[\exp \left(-\lambda_{\text {Gyro }} \cdot t\right)\right.
$$$$
+\rho_{\text {Gyro }}(t) \cdot \exp \left(-\lambda_{\text {Gyro }} . t\right)
$$$$
\left.\cdot \sum_{j=1}^{N_{\text {Gyro }}-1} \frac{\left(\lambda_{\text {Gyro }} \cdot t\right)^{j}}{j !}\right] \times\left[\exp \left(-\lambda_{S S} \cdot t\right)^{N_{S S}}\right]
$$$$
\times\left[\exp \left(-\lambda_{M T} \cdot t\right)^{N_{M T}}\right]
$$$$
\times\left[\sum_{k=2}^{N_{R W}}\left(\begin{array}{c}
N_{R W} \\
k
\end{array}\right) \exp \left(-\lambda_{R W} \cdot t\right)^{k}\right.
$$$$
\left.\left(1-\exp \left(-\lambda_{R W} \cdot t\right)\right)^{N_{R W}-k}\right],
$$

$R_{S E}(t)=\left[\exp \left(-\lambda_{E C U} \cdot t\right)+\rho_{E C U}(t) \cdot \exp \left(-\lambda_{E C U} \cdot t\right)\right.$

$$
\begin{aligned}
& \left.\cdot \sum_{j=1}^{N_{E C U}-1} \frac{\left(\lambda_{E C U} \cdot t\right)^{j}}{j !}\right] \times \\
& {\left[1-\left(1-\exp \left(-\lambda_{\text {Int }} \cdot t\right)\right)^{N_{I n t}}\right]} \\
& \times\left[\exp \left(-\lambda_{M M} \cdot t\right)+\rho_{M M}(t) \cdot \exp \left(-\lambda_{M M} \cdot t\right)\right.
\end{aligned}
$$

$$
\begin{aligned}
& \left.\cdot \sum_{j=1}^{N_{M M}-1} \frac{\left(\lambda_{M M} \cdot t\right)^{j}}{j !}\right] \times\left[\exp \left(-\lambda_{S S} \cdot t\right)^{N_{S S}}\right] \\
& \times\left[\exp \left(-\lambda_{M T} \cdot t\right)^{N_{M T}}\right]
\end{aligned}
$$

$C_{E C U} \cdot N_{E C U}+C_{\text {Int }} \cdot N_{\text {Int }}+C_{M M} \cdot N_{M M}$

$+C_{\text {Gyro }} . N_{\text {Gyro }}+C_{S T} \cdot N_{S T}$

$+C_{R W} \cdot N_{R W} D_{E C U} \cdot x_{E C U}+D_{\text {Int }} \cdot x_{I n t}$

$+D_{M M} \cdot x_{M M}+D_{\text {Gyro }} \cdot x_{G y r o}+D_{S S} \cdot x_{S S}$

$+D_{S T} \cdot x_{S T}+D_{M T} \cdot x_{M T}+D_{R W} \cdot x_{R W} \leq C_{\max }$,

$W_{E C U} \cdot N_{E C U}+W_{I n t} \cdot N_{I n t}+W_{M M} \cdot N_{M M}$

$$
\begin{aligned}
& +W_{\text {Gyro }} \cdot N_{\text {Gyro }}+W_{S T} \cdot N_{S T} \\
& +W_{R W} \cdot N_{R W} \leq W_{\max }
\end{aligned}
$$

$L_{E C U} \leq N_{E C U} \leq U_{E C U}$,

$L_{\text {Int }} \leq N_{\text {Int }} \leq U_{\text {Int }}$,

$L_{M M} \leq N_{M M} \leq U_{M M}$,

$L_{\text {Gyro }} \leq N_{\text {Gyro }} \leq U_{\text {Gyro }}$,

$L_{S T} \leq N_{S T} \leq U_{S T}$,

$L_{R W} \leq N_{R W} \leq U_{R W}$,

$\lambda_{E C U}=\lambda_{E C U}^{\max }\left(1-\frac{x_{E C U}}{100}\right)$,

$\lambda_{\text {Int }}=\lambda_{\text {Int }}^{\max }\left(1-\frac{x_{\text {Int }}}{100}\right)$,

$\lambda_{M M}=\lambda_{M M}^{\max }\left(1-\frac{x_{M M}}{100}\right)$,

$\lambda_{M M}=\lambda_{M M}^{\max }\left(1-\frac{x_{M M}}{100}\right)$,

$\lambda_{S S}=\lambda_{S S}^{\max }\left(1-\frac{x_{S S}}{100}\right)$,

$\lambda_{S T}=\lambda_{S T}^{\max }\left(1-\frac{x_{S T}}{100}\right)$,

$\lambda_{M T}=\lambda_{M T}^{\max }\left(1-\frac{x_{M T}}{100}\right)$,

$\lambda_{R W}=\lambda_{R W}^{\max }\left(1-\frac{x_{R W}}{100}\right)$, 


$$
\begin{aligned}
& \lambda_{E C U}^{\min } \leq \lambda_{E C U} \leq \lambda_{E C U}^{\max }, \\
& \lambda_{\text {Int }}^{\min } \leq \lambda_{\text {Int }} \leq \lambda_{\text {Int }}^{\max }, \\
& \lambda_{M M}^{\min } \leq \lambda_{M M} \leq \lambda_{M M}^{\max }, \\
& \lambda_{G y r o}^{\min } \leq \lambda_{G y r o} \leq \lambda_{G y r o}^{\max }, \\
& \lambda_{S S}^{\min } \leq \lambda_{S S} \leq \lambda_{S S}^{\max }, \\
& \lambda_{S T}^{\min } \leq \lambda_{S T} \leq \lambda_{S T}^{\max }, \\
& \lambda_{M T}^{\min } \leq \lambda_{M T} \leq \lambda_{M T}^{\max }, \\
& \lambda_{R W}^{\min } \leq \lambda_{R W} \leq \lambda_{R W}^{\max }, \\
& x_{E C U}, x_{\text {Int }}, x_{M M}, x_{G y r o}, x_{S S}, x_{S T}, x_{M T}, x_{R W} \\
& \quad \in \text { Integer, }
\end{aligned}
$$

$N_{E C U}, N_{\text {Int }}, N_{M M}, N_{G y r o}, N_{S T}, N_{R W} \in$ Integer

$\lambda_{E C U}, \lambda_{\text {Int }}, \lambda_{M M}, \lambda_{\text {Gyro }}, \lambda_{S S}, \lambda_{S T}, \lambda_{M T}, \lambda_{R W} \geq 0$.

The objective function of the above mathematical model (6) is to maximize the minimum reliability of the attitude determination and control system under different functional modes. Constraints (7) to (11) calculate system reliability in the functional modes of de tumbling, coarse pointing, fine pointing, sun pointing, and safe, respectively. Constraint (12) guarantees that the costs of redundancy allocation be included in the total costs incurred on the system and that the failure rate improvement does not exceed the maximum allowable cost. Constraint (13) ensures that the weight of the components allocated to the system does not exceed the maximum allowable weight specified for it. Constraints (14) to (19) ensure that the allocation of redundant components to the system does not exceed the minimum and maximum allowable values specified for each component. Constraints (20) to (27) are a set of calculative constraints that calculate the failure rate of each component based on the maximum possible failure rate and the percentage of improvement in reducing it. Constraints (28) to (35) ensure that the failure rate of each component does not exceed the minimum and maximum values specified for them.

\section{Solution procedure}

The mathematical optimization model presented in this study falls into the category of optimization problems associated with Mixed Integer Nonlinear Programming (MINLP). In most cases, on the other hand, the issue of over-allocation in terms of computational time falls

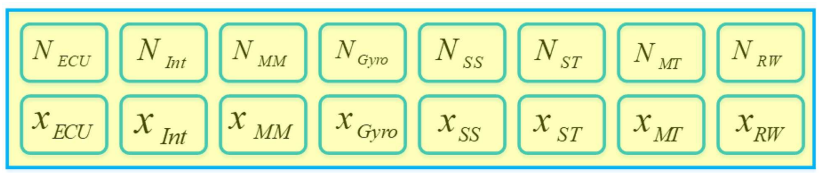

Figure 8. Chromosome structure.

into the category of NP-HARD problems. Therefore, metaheuristic algorithms should be used to solve the proposed mathematical model. In this section, a genetic algorithm is used to solve the proposed model.

The general form of the solutions related to the presented mathematical model consists of a matrix. The main focus of this research to solve the problem is on the genetic algorithm, to be explained and interpreted in the following search operators. The structure of the solution presented in this study, according to Figure 8, consists of a two-row matrix. In the first row, the number of redundant components in the system is specified; in the second row, the percentage of improvement in reducing the failure rate of each component relative to the maximum failure rate is determined.

After generating the chromosome, evaluation of each chromosome is calculated. For this purpose, the values of $Z$, Cost, and Weight are calculated using the presented mathematical model. Then, the evaluation of each solution is determined as in Eq. (37):

$f=$

$$
\frac{z}{1+\max \left(\operatorname{Cos} t-C_{\max }, 0\right)+\max \left(\text { Weight }-W_{\max }, 0\right)},
$$

where $Z$, Cost, and Weight functions are calculated through Eqs. (38), (39), and (40). Thus, solutions that do not meet the maximum budget and maximum weight constraints will be penalized.

$$
\begin{aligned}
& Z=\max \left\{\min \left\{R_{D}, R_{C}, R_{F}, R_{S U}, R_{S E}\right\}\right\} \\
& \text { Cost }= C_{E C U} \cdot N_{E C U}+C_{\text {Int }} \cdot N_{I n t}+C_{M M} \cdot N_{M M} \\
&+C_{G y r o} \cdot N_{G y r o}+C_{S T} \cdot N_{S T} \\
&+C_{R W} \cdot N_{R W} D_{E C U} \cdot x_{E C U}+D_{\text {Int }} \cdot x_{I n t} \\
&+D_{M M} \cdot x_{M M}+D_{G y r o} \cdot x_{G y r o}+D_{S S} \cdot x_{S S} \\
&+D_{S T} \cdot x_{S T}+D_{M T} \cdot x_{M T}+D_{R W} \cdot x_{R W},
\end{aligned}
$$

$$
\begin{aligned}
\text { Weight }= & W_{E C U} \cdot N_{E C U}+W_{\text {Int }} \cdot N_{\text {Int }}+W_{M M} \cdot N_{M M} \\
& +W_{\text {Gyro }} \cdot N_{\text {Gyro }}+W_{S S} \cdot N_{S S}+W_{S T} \cdot N_{S T} \\
& +W_{M T} \cdot N_{M T}+W_{R W} \cdot N_{R W} .
\end{aligned}
$$

In this research, the roulette wheel mechanism has been used for the selection strategy. Holland [29] first suggested choosing a roulette wheel. 


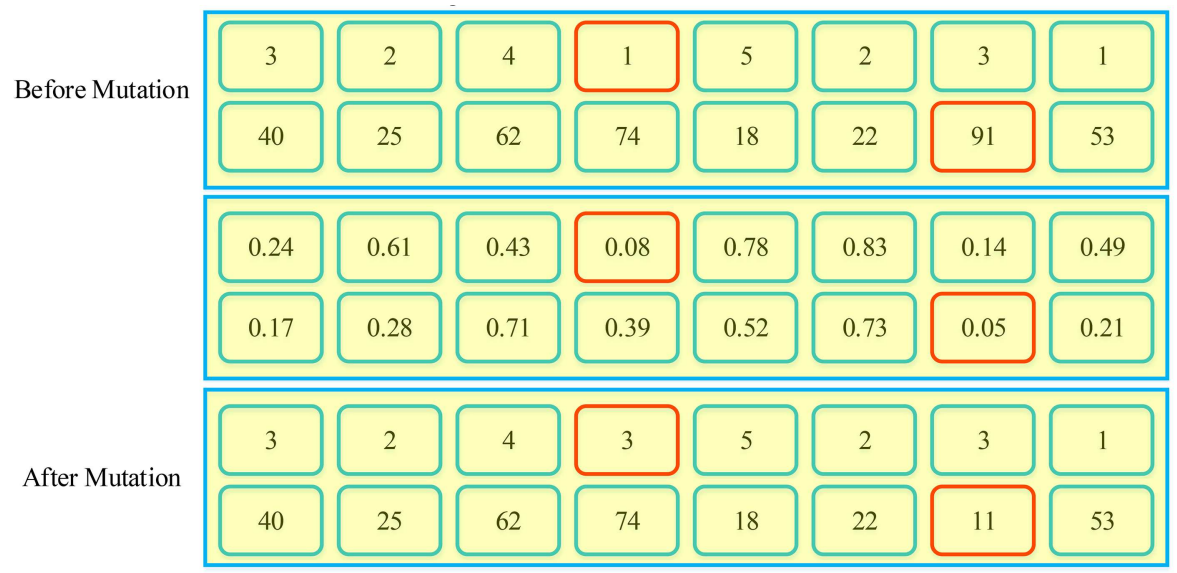

Figure 9. An example of the mutation operator.

Parents are first selected to perform the crossover operator and then, the children are generated using a uniform crossover operator. The operation of this operator is described in [30,31]. In this operator, for each gene in the selected parent chromosome, a number between zero and one is randomly generated; then, the children chromosomes are quantified in linear composition of the parent chromosomes.

Mutations are also performed on each array of the chromosome matrix. In this operator, after selecting the desired parent, a random number between zero and one is generated for each gene in the parent chromosome, and the values of the parent chromosome genes are mutated at a certain rate of mutation. Now, if the generated random number is less than the desired mutation rate, the corresponding gene on the parent chromosome will be randomly mutated. However, if the random number generated is larger than the mutation rate, the gene will not be mutated on the parent chromosome [31]. For example, Figure 9 shows how the mutation operator operates on a chromosome.

The application of a Random Search (RS) method to solve the presented model can be a lower bound for minimization problems and a lower bound for maximization problems compared to other solution methods. In fact, proving the intelligent performance of meta-heuristic algorithms can be demonstrated by comparing them to an RS. Therefore, these algorithms must always be more powerful than an RS. An RS is provided to validate the proposed algorithm. The proposed RS pseudocode is shown in Figure 10 [32].

\section{Results and discussions}

In this section, the proposed model is solved. For this purpose, first, a numerical example is presented in the case study. Table 4 shows the parameters and the information needed to solve the problem for a numerical sample example.
Initialize $\boldsymbol{X}$ with a random position in the search space. Until a termination criterion (number of iterations) is met, repeat the following:

Sample a new position $\boldsymbol{y}$ from the hyper sphere of a given radius surrounding the current position $\boldsymbol{x}$ If $(f(\boldsymbol{y})<f(\boldsymbol{x}))$ then move to the new position by setting $\boldsymbol{x}=\boldsymbol{y}$

Now $\boldsymbol{x}$ holds the best-found position.

Figure 10. Pseudocode of random search method.

Table 4. Parameters in the numerical example.

\begin{tabular}{cccccc}
\hline$t$ & 100 & $U_{E C U}$ & 9 & $C_{G y r o}$ & 30 \\
\hline$\rho_{E C U}$ & 0.99 & $L_{E C U}$ & 2 & $W_{\text {Gyro }}$ & 30 \\
$\rho_{M M}$ & 0.99 & $U_{\text {Int }}$ & 8 & $D_{\text {Gyro }}$ & 50 \\
$\rho_{G y r o}$ & 0.99 & $L_{\text {Int }}$ & 2 & $D_{S S}$ & 50 \\
$\rho_{S T}$ & 0.99 & $U_{M M}$ & 10 & $C_{S T}$ & 5 \\
$\lambda_{E C U}^{\max }$ & 0.005 & $L_{M M}$ & 2 & $W_{S T}$ & 20 \\
$\lambda_{E C U}^{\min }$ & 0.0001 & $U_{G y r o}$ & 10 & $D_{S T}$ & 50 \\
$\lambda_{I n t}^{\max }$ & 0.003 & $L_{G y r o}$ & 2 & $D_{M T}$ & 50 \\
$\lambda_{I n t}^{\min }$ & 0.0002 & $U_{S T}$ & 8 & $C_{R W}$ & 10 \\
$\lambda_{M M}^{\max }$ & 0.004 & $L_{S T}$ & 2 & $W_{R W}$ & 25 \\
$\lambda_{M M}^{\min }$ & 0.0005 & $U_{R W}$ & 11 & $D_{R W}$ & 50 \\
$\lambda_{G y r o}^{\max }$ & 0.006 & $L_{R W}$ & 4 & $C_{\max }$ & 35000 \\
$\lambda_{G y r o}^{\min }$ & 0.0003 & $C_{E C U}$ & 10 & $W_{\max }$ & 1000 \\
$\lambda_{S S}^{\max }$ & 0.007 & $W_{E C U}$ & 20 & $N_{S S}$ & 6 \\
$\lambda_{S S}^{\min }$ & 0.0002 & $D_{E C U}$ & 50 & $N_{M T}$ & 3 \\
$\lambda_{S T}^{\max }$ & 0.001 & $C_{I n t}$ & 20 & & \\
$\lambda_{S T}^{\min }$ & 0.0001 & $W_{I n t}$ & 25 & & \\
$\lambda_{M T}^{\max }$ & 0.002 & $D_{I n t}$ & 50 & & \\
$\lambda_{M T}^{\min }$ & 0.0001 & $C_{M M}$ & 15 & & \\
$\lambda_{R W}^{\max }$ & 0.005 & $W_{M M}$ & 40 & & \\
$\lambda_{R W}^{\min }$ & 0.0002 & $D_{M M}$ & 50 & & \\
\hline & & & & & \\
\hline
\end{tabular}


Table 5. Interval search Parameters levels of genetic algorithm.

\begin{tabular}{cccc}
\hline Parameter & Interval & $\begin{array}{c}\text { Lower } \\
\text { bound }\end{array}$ & $\begin{array}{c}\text { Upper } \\
\text { bound }\end{array}$ \\
\hline$N p o p$ & {$[50-100]$} & 50 & 100 \\
$P_{c}$ & {$[0.4-0.7]$} & 0.4 & 0.7 \\
$P_{m}$ & {$[0.1-0.3]$} & 0.1 & 0.3 \\
\hline
\end{tabular}

To solve the above example, the parameters of the genetic algorithm must first be tuned. The objective of tuning the input parameters of algorithms is to achieve appropriate criteria for the objective function of the algorithm. The result of meta-heuristic algorithms depends on the values of their input parameters. Therefore, we explain in detail how to set the values of these parameters. Input parameters of the genetic algorithm are population size $(N p o p)$, crossover rate $\left(P_{c}\right)$, and mutation rate $\left(P_{m}\right)$. Each of these parameters is of particular importance and affects the performance of this algorithm. In order to recognize the appropriate values of the parameters such that the criterion of the objective function leads to appropriate solutions, the Response Surface Methodology (RSM) technique was used [33]. The main parameters of this algorithm are considered in Table 5 so that the appropriate levels are tuned. To select the two-level experimental factor design, each of the experiments is considered at two levels: high and low. The method for advancing the RSM should be implemented in such a way that in addition to the upper and lower limits, the axial points using the middle limits as well as a number of central points (in this research, 5 central points are added to the design) are also considered. For this algorithm, according to the three available parameters, $2^{3}$ factor is considered. To this end, the experiment in MINITAB 16 software is performed for the algorithm and the best level is tuned for the test result.

According to the above explanations, the nonlinear regression equation for the proposed genetic algorithm, which shows the relationship between the parameters of the algorithm and the value of the objective function, is obtained. Now, it is enough to solve Model (41) to get the optimal parameters of the genetic algorithm.

$$
\begin{aligned}
\operatorname{Max} & 0.875248-0.000396038 \times N \text { pop }-0.0368956 \\
& \times P_{c}-0.0567818 \times P_{m}+3.03127 e-006 \\
& \times N p o p \times N p o p+0.0348687 \times P_{c} \times P_{c} \\
& -0.189545 \times P_{m} \times P_{m}+0.000768 \times N p o p \\
& \times P_{m}-0.000241333 \times N p o p \times P_{c}+0.128 \\
& \times P_{m} \times P_{c}
\end{aligned}
$$

Table 6. Optimal value of genetic algorithm parameters.

\begin{tabular}{cc}
\hline Parameter & Optimal value \\
\hline$N$ pop & 100 \\
$P_{c}$ & 0.7 \\
$P_{m}$ & 0.2891614 \\
\hline
\end{tabular}

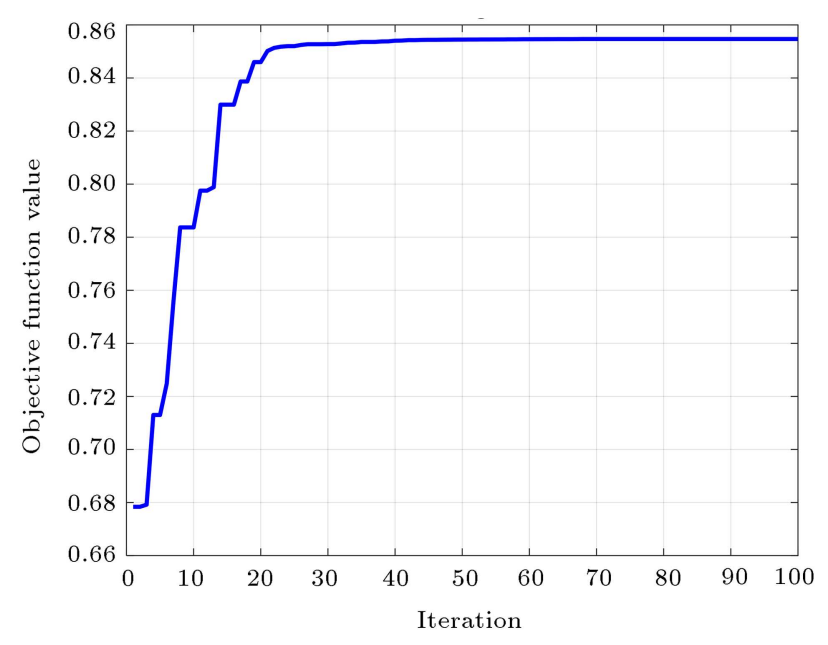

Figure 11. Convergence diagram of genetic algorithm.

$$
\begin{aligned}
& \text { s.t.: } \\
& 50 \leq N \text { pop } \leq 100, \\
& 0.4 \leq P_{c} \leq 0.7, \\
& 0.1 \leq P_{c} \leq 0.3 \\
& N p o p \in Z \text {. }
\end{aligned}
$$

Solving the above model in Lingo software determined the values of the parameters of the genetic algorithm, as shown in Table 6 .

After obtaining the tuned parameters of the genetic algorithm, the example presented in Table 4 is solved using the genetic algorithm developed in this study and its results are reported. As shown in Figure 11, the convergence diagram of the genetic algorithm is shown in consecutive iterations. In addition, as shown in Tables 7 and 8, the information about the variables of the mathematical optimization model provided under the available maximum budget is clearly presented.

\section{Sensitivity analysis and validation}

In this section, in order to validate the genetic algorithm developed to solve the mathematical model, which was discussed in Sections 4-6, the results of this algorithm were compared with the solutions obtained from the RS method under different amounts of the maximum budgets. Given that the solutions 
Table 7. The variables of the mathematical optimization model provided by GA under $C \max =35000$.

\begin{tabular}{cccccc}
\hline$R_{D}(t)$ & 0.9696 & $\lambda_{\text {Int }}$ & 0.0019 & $x_{S S}$ & 97 \\
$R_{C}(t)$ & 0.8548 & $x_{\text {Int }}$ & 37 & $N_{S T}$ & 4 \\
$R_{F}(t)$ & 0.8547 & $N_{M M}$ & 6 & $\lambda_{S T}$ & 0.0001 \\
$R_{S U}(t)$ & 0.8548 & $\lambda_{M M}$ & 0.00052 & $x_{S T}$ & 90 \\
$R_{S E}(t)$ & 0.8550 & $x_{M M}$ & 87 & $\lambda_{M T}$ & 0.0001 \\
$N_{E C U}$ & 5 & $N_{\text {Gyro }}$ & 5 & $x_{M T}$ & 95 \\
$\lambda_{E C U}$ & 0.0001 & $\lambda_{\text {Gyro }}$ & 0.0003 & $N_{R W}$ & 9 \\
$x_{E C U}$ & 98 & $x_{\text {Gyro }}$ & 95 & $\lambda_{R W}$ & 0.0006 \\
$N_{\text {Int }}$ & 8 & $\lambda_{S S}$ & 0.00021 & $x_{R W}$ & 88 \\
\hline & & & & & \\
\hline
\end{tabular}

Table 8. The variables of the mathematical optimization model provided by GA under $C \max =5000$.

\begin{tabular}{cccccc}
\hline$R_{D}(t)$ & 0.5410 & $\lambda_{\text {Int }}$ & 0.003 & $x_{S S}$ & 90 \\
$R_{C}(t)$ & 0.3553 & $x_{\text {Int }}$ & 0 & $N_{S T}$ & 6 \\
$R_{F}(t)$ & 0.3533 & $N_{M M}$ & 4 & $\lambda_{S T}$ & 0.001 \\
$R_{S U}(t)$ & 0.3553 & $\lambda_{M M}$ & 0.004 & $x_{S T}$ & 0 \\
$R_{S E}(t)$ & 0.3572 & $x_{M M}$ & 0 & $\lambda_{M T}$ & 0.002 \\
$N_{E C U}$ & 5 & $N_{\text {Gyro }}$ & 5 & $x_{M T}$ & 0 \\
$\lambda_{E C U}$ & 0.005 & $\lambda_{\text {Gyro }}$ & 0.006 & $N_{R W}$ & 11 \\
$x_{E C U}$ & 0 & $x_{\text {Gyro }}$ & 0 & $\lambda_{R W}$ & 0.005 \\
$N_{I n t}$ & 5 & $\lambda_{S S}$ & 0.0007 & $x_{R W}$ & 0 \\
\hline
\end{tabular}

Table 9. Comparison of the results obtained from the genetic algorithm and random search to solve the problem presented under different values of the maximum budget available.

\begin{tabular}{ccc}
\hline $\boldsymbol{C}_{\max }$ & Genetic algorithm & Random search \\
\hline 5000 & 0.3533 & - \\
7500 & 0.6075 & 0.0468 \\
10000 & 0.8248 & 0.1588 \\
12500 & 0.8443 & 0.4527 \\
15000 & 0.8461 & 0.5792 \\
17500 & 0.8484 & 0.7001 \\
20000 & 0.8501 & 0.7923 \\
22500 & 0.8516 & 0.7793 \\
25000 & 0.8524 & 0.7996 \\
27500 & 0.8540 & 0.8438 \\
30000 & 0.8543 & 0.8177 \\
32500 & 0.8546 & 0.8249 \\
35000 & 0.8547 & 0.8331 \\
\hline
\end{tabular}

obtained from the RS method are always far from global optimization, these solutions represent good criteria for evaluating the performance of the genetic algorithm. As can be seen in Table 9, genetics in all cases offers better solutions than RS, implying the efficient operation of this algorithm to solve the current

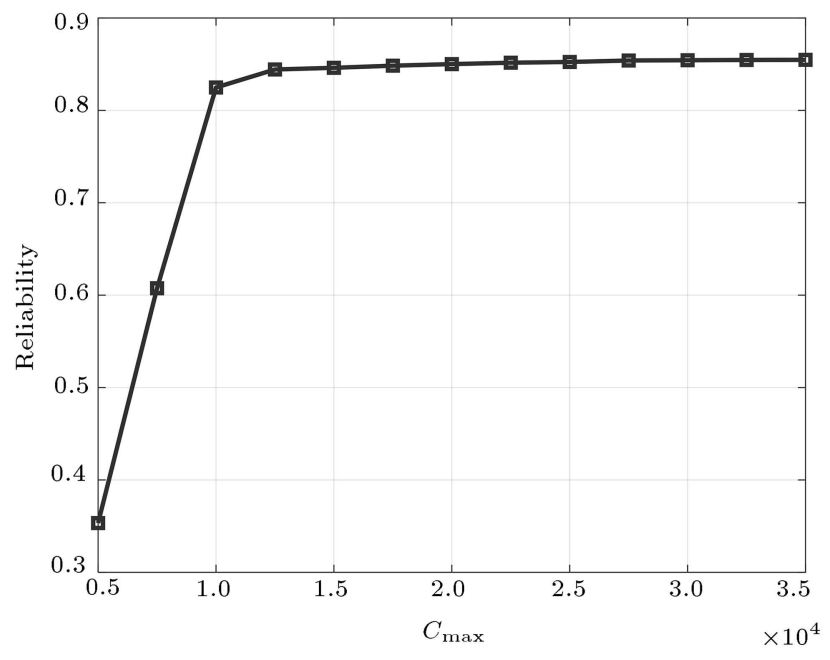

Figure 12. Sensitivity analysis of the results obtained from the genetic algorithm to solve the problem presented under different values of the maximum budget available.

problem. Figure 12 shows the effect of increasing the available maximum budget on the reliability of the system under study. It is worth mentioning that the 2018 version of MATLAB software was used in this paper to implement the RS method and genetic algorithm.

\section{Conclusions}

This study introduced the satellite attitude determination and control system as well as the structure of the studied system and its components. Then, the reliability of this system was modeled and optimized using the mathematical approach based on the combination of reliability allocation and redundancy allocation. As demonstrated through the analysis of this system, in general, the satellite attitude determination and control system was found to be having five different functional modes. These modes included de tumbling, coarse pointing, fine pointing, sun pointing, and safe. In the following, each of these functional modes was introduced and the reliability block diagram of the system was presented under each of these modes. In this system, the function of the components used in the system and the components failure were independent of each other. Failed components did not damage the system as a whole. Moreover, the components were binary. In the attitude determination and control system, components could not be repaired and returned to the system after failure. In the mathematical optimization model presented in this study, the parameters related to the cost and weight of the components in the system were deterministic and definite, and the system had the maximum cost and maximum allowable weight for the components used in it. The different subsystems of the system under study have active, cold-ready, 
or $k$-out-of- $n$ strategies, which are identified in the reliability block diagram of each mode. Also, in the case of systems whose components are cold-standby, there was a possibility that the switch would fail. In this study, the failure rate of the components used in the system was constant; thus, the life distribution of the components used in the system was exponential. In this study, the percentage of improvements made to the failure rates of the components was integer and the failure rate of the components used in the closed continuous interval could be changed. The mathematical optimization model presented in this study fell into the category of optimization problems related to Mixed Integer Nonlinear Programming (MINLP). Solving these problems always involves much mathematical complexity. Therefore, the development of exact solution methods for these problems is very difficult and, in most cases, impossible. On the other hand, the redundancy allocation problem in terms of computational time fell into the class of NP-HARD problems. Therefore, metaheuristic algorithms should be used to solve the proposed mathematical model. In this study, a genetic algorithm was used to solve the proposed model. Also, to validate the results obtained from the developed genetic algorithm, a random search algorithm was used in which, according to the obtained outputs, the genetic algorithm had significantly better performance in all cases than random search. In this study, in order to tune the parameters of the genetic algorithm, the response surface methodology was used based on which the parameters of the number of population in each iteration, crossover rate, and mutation rate were tuned.

\section{References}

1. Kim, D.S., Lee, S.M., Jung, J.H., et al. "Reliability and availability analysis for an on board computer in a satellite system using standby redundancy and rejuvenation", Journal of Mechanical Science and Technology, 26(7), pp. 2059-2063 (2012).

2. Lakshminarayana, V., Karthikeyan, B., Hariharan, V.K., et al. "Impact of space weather on spacecraft", In 2008 10th International Conference on Electromagnetic Interference \& Compatibility, pp. 481-486 (2008).

3. Poulsen, K. "Satellites at risk of hacks", Security Focus (2002).

4. Huang, Y., Kintala, C., Kolettis, N., et al. "Software rejuvenation: Analysis, module and applications", In Twenty-Fifth International Symposium on FaultTolerant Computing, pp. 381-390 (1995).

5. Castet, J.F. and Saleh, J.H. "Satellite and satellite subsystems reliability: Statistical data analysis and modeling", Reliability Engineering \& System Safety, 94(11), pp. 1718-1728 (2009).
6. Castet, J.F. and Saleh, J.H. "Beyond reliability, multistate failure analysis of satellite subsystems: a statistical approach", Reliability Engineering \& System Safety, 95(4), pp. 311-322 (2010).

7. Nagiya, K. and Ram, M. "Reliability characteristics of a satellite communication system including earth station and terrestrial system", International Journal of Performability Engineering, 9(6), pp. 667-676 (2013).

8. Fyffe, D.E., Hines, W.W., and Lee, N.K. "System reliability allocation and a computational algorithm", IEEE Transactions on Reliability, 17(2), pp. 64-69 (1968).

9. Nakagawa, Y. and Miyazaki, S. "Surrogate constraints algorithm for reliability optimization problems with two constraints", IEEE Transactions on Reliability, 30(2), pp. 175-180 (1981).

10. Garg, H. and Sharma, S.P. "Multi-objective reliabilityredundancy allocation problem using particle swarm optimization", Computers \& Industrial Engineering, 64(1), pp. 247-255 (2013).

11. Khalili-Damghani, K., Abtahi, A.R., and Tavana, M. "A decision support system for solving multi-objective redundancy allocation problems", Quality and Reliability Engineering International, 30(8), pp. 1249-1262 (2014).

12. Chambari, A., Najafi, A.A., Rahmati, S.H.A., et al. "An efficient simulated annealing algorithm for the redundancy allocation problem with a choice of redundancy strategies", Reliability Engineering \& System Safety, 119, pp. 158-164 (2013).

13. Gago, J., Hartillo, I., Puerto, J., et al. "Exact cost minimization of a series-parallel reliable system with multiple component choices using an algebraic method", Computers \& Operations Research, 40(11), pp. 2752-2759 (2013).

14. Ebrahimipour, V., Asadzadeh, S.M., and Azadeh, A. "An emotional learning-based fuzzy inference system for improvement of system reliability evaluation in redundancy allocation problem", The International Journal of Advanced Manufacturing Technology, 66(912), pp. 1657-1672 (2013).

15. Liu, Y., Huang, H.Z., Wang, Z., et al. "A joint redundancy and imperfect maintenance strategy optimization for multi-state systems", IEEE Transactions on Reliability, 62(2), pp. 368-378 (2013).

16. Ding, Y. and Lisnianski, A. "Fuzzy universal generating functions for multi-state system reliability assessment", Fuzzy Sets and Systems, 159(3), pp. 307-324 (2008).

17. Ouzineb, M., Nourelfath, M., and Gendreau, M. "A heuristic method for non-homogeneous redundancy optimization of series-parallel multi-state systems", Journal of Heuristics, 17(1), pp. 1-22 (2011).

18. Sharma, V.K. and Agarwal, M. "Ant colony optimization approach to heterogeneous redundancy in multistate systems with multi-state components", In 2009 8th International Conference on Reliability, Maintainability and Safety, pp. 116-121 (2009). 
19. Ouzineb, M., Nourelfath, M., and Gendreau, M. "Tabu search for the redundancy allocation problem of homogenous series-parallel multi-state systems", Reliability Engineering \& System Safety, 93(8), pp. 1257-1272 (2008).

20. Levitin, G., Xing, L., Ben-Haim, H., et al. "Reliability of series-parallel systems with random failure propagation time", IEEE Transactions on Reliability, 62(3), pp. $637-647$ (2013).

21. Lins, I.D. and Droguett, E.L. "Redundancy allocation problems considering systems with imperfect repairs using multi-objective genetic algorithms and discrete event simulation", Simulation Modelling Practice and Theory, 19(1), pp. 362-381 (2011).

22. Lins, I.D. and Droguett, E.L. "Multiobjective optimization of availability and cost in repairable systems design via genetic algorithms and discrete event simulation", Pesquisa Operacional, 29(1), pp. 43-66 (2009).

23. Maatouk, I., Chătelet, E., and Chebbo, N. "Availability maximization and cost study in multi-state systems", In 2013 Proceedings Annual Reliability and Maintainability Symposium (RAMS), pp. 1-6 (2013).

24. Garg, H., Rani, M., and Sharma, S.P. "An efficient two phase approach for solving reliability-redundancy allocation problem using artificial bee colony technique", Computers \& Operations Research, 40(12), pp. 29612969 (2013).

25. Ebrahimipour, V. and Sheikhalishahi, M. "Application of multi-objective particle swarm optimization to solve a fuzzy multi-objective reliability redundancy allocation problem", In 2011 IEEE International Systems Conference, pp. 326-333 (2011).

26. Miriha, M., Niaki, S.T.A., Karimi, B., et al. "Biobjective reliability optimization of switch-mode $\mathrm{k}$ out-of-n series-parallel systems with active and cold standby components having failure rates dependent on the number of components", Arabian Journal for Science and Engineering, 42(12), pp. 5305-5320 (2017).

27. Mousavi, S.M., Alikar, N., Niaki, S.T.A., et al. "Two tuned multi-objective meta-heuristic algorithms for solving a fuzzy multi-state redundancy allocation problem under discount strategies", Applied Mathematical Modelling, 39(22), pp. 6968-6989 (2015)

28. Zaretalab, A., Hajipour, V., Sharifi, M., et al. "A knowledge-based archive multi-objective simulated annealing algorithm to optimize series-parallel system with choice of redundancy strategies", Computers \& Industrial Engineering, 80, pp. 33-44 (2015).

29. Holland, J.H., Adaptation in Natural and Artificial Systems: An Introductory Analysis with Applications to Biology, Control, and Artificial Intelligence, MIT press (1992).

30. Gen, M., Cheng, R., and Wang, D. "Genetic algorithms for solving shortest path problems", In Proceedings of 1997 IEEE International Conference on Evolutionary Computation (ICEC'97), pp. 401-406 (1997).

31. Tavakkoli-Moghaddam, R., Safari, J., and Sassani, F. "Reliability optimization of series-parallel systems with a choice of redundancy strategies using a genetic algorithm", Reliability Engineering \& System Safety, 93(4), pp. 550-556 (2008).

32. Rastrigin, L.A. "The convergence of the random search method in the extremal control of a many parameter system", Automaton and Remote Control, 24, pp. 1337-1342 (1963).

33. Montgomery, D.C., Design and Analysis of Experiments, John Wiely and Sons (2017).

\section{Biographies}

Akbar Mansouri is a $\mathrm{PhD}$ candidate at the Department of Industrial Engineering at Islamic Azad University, Qazvin Branch (QIAU) in Iran. He received his BSc and MSc degrees in Industrial Engineering from Amirkabir University of technology (Tehran Polytechnic) in Iran. His research interests include reliability engineering, combinatorial optimization, multiobjective optimization, computational intelligence, and data mining.

Akbar Alem-Tabriz received his $\mathrm{PhD}$ degree in Management from Turkey in 1989. He is currently an Associated Professor at Shahid Beheshti University. His research interests are performance evaluation in production industries, implementation of total quality management, and efficiency and productivity measurement in Industry. He has published several papers in national and international journals. 